\title{
Extended genetic analysis of Brazilian isolates of Bacillus cereus and Bacillus thuringiensis
}

\author{
Viviane Zahner ${ }^{1 /+}$, Ana Carolina Telles de Carvalho e Silva ${ }^{1}$, \\ Gabriela Pinhel de Moraes', Douglas McIntosh', Ivano de Filippis²
}

\begin{abstract}
${ }^{1}$ Laboratório de Transmissores de Leishmanioses, Setor de Entomologia Médica Forense, Instituto Oswaldo Cruz ${ }^{2}$ Departamento de Microbiologia, Instituto Nacional de Controle de Qualidade em Saúde-Fiocruz, Rio de Janeiro, RJ, Brasil

${ }^{3}$ Departamento de Parasitologia Animal, Universidade Federal Rural do Rio de Janeiro, Rio de Janeiro, RJ, Brasil
\end{abstract}

Multiple locus sequence typing (MLST) was undertaken to extend the genetic characterization of 29 isolates of Bacillus cereus and Bacillus thuringiensis previously characterized in terms of presencelabsence of sequences encoding virulence factors and via variable number tandem repeat (VNTR). Additional analysis involved polymerase chain reaction for the presence of sequences (be, cytK, in A, pag, lef, cya and cap), encoding putative virulence factors, not investigated in the earlier study. MLST analysis ascribed novel and unique sequence types to each of the isolates. A phylogenetic tree was constructed from a single sequence of 2,838 bp of concatenated loci sequences. The strains were not monophyletic by analysis of any specific housekeeping gene or virulence characteristic. No clear association in relation to source of isolation or to genotypic profile based on the presence or absence of putative virulence genes could be identified. Comparison of VNTR profiling with MLST data suggested a correlation between these two methods of genetic analysis. In common with the majority of previous studies, MLST was unable to provide clarification of the basis for pathogenicity among members of the B. cereus complex. Nevertheless, our application of MLST served to reinforce the notion that $\mathrm{B}$. cereus and $\mathrm{B}$. thuringiensis should be considered as the same species.

Key words: Bacillus cereus - multiple locus sequence typing - virulence factors

The Bacillus cereus group comprises six valid species, including B. cereus, Bacillus thuringiensis, Bacillus anthracis and Bacillus mycoides that are distinguished based on plasmid content and the expression of morphological characteristics that confer distinctive pathogenicity features. These species are highly related and in the opinion of many researchers they may be considered as a single species based on genetic and genomic features (Helgason et al. 2000a, b, Anderson et al. 2005, Zahner et al. 2005, Han et al. 2006). B. cereus and B. thuringiensis are frequently found as components of the normal microflora of the gastrointestinal tract of a variety of mammal and insects (Jensen et al. 2003). However, the importance of this group of bacteria is related to food safety, their role as mammalian and/or insect pathogens and their application as biological control agents. B. cereus is an opportunistic human pathogen involved primarily in food poisoning incidents and increasingly as a source of nosocomial infections, while $B$. thuringiensis is one of the world's most widely used biopesticides. Although the symptoms of $B$. cereus food-borne illness are relatively mild, severe cases have been reported, including death by cereulide toxin (emetic toxin) and necrotic enterotoxin, cytotoxin CytK producing strains (Mahler et al. 1997,

Financial support: FAPERJ, CNPq, CIEE

+ Corresponding author: vzahner@ioc.fiocruz.br

Received 10 April 2012

Accepted 17 July 2012
Lund et al. 2000). The heterogeneity among strains of $B$. cereus in the context of virulence is also a point of study. In this context, $B$. cereus strains have been shown to harbour diverse plasmids which shared conserved sequences with the $B$. anthracis virulence plasmids $\mathrm{pXO} 1$ and $\mathrm{pXO} 2$ (Jackson et al. 1998), an example is strain G9241, responsible for a case of severe pneumonia, that contained the plasmid pBCX01 which was $99.6 \%$ similar to the $B$. anthracis $\mathrm{pXO1}$ virulence plasmid (Hoffmaster et al. 2004). Moreover, sequences encoding toxins or capsular polysaccharide of $B$. anthracis (pagA, lef, cya and capBCA) were detected in several $B$. cereus strains recovered from patients (not known to be immune compromised) with fatal pneumonia (Jackson et al. 1998).

In Brazil, the isolation of B. cereus from food is not infrequent, but food poisoning is not commonly reported. In a previous study the presence of a variety of sequences (bce $\mathrm{T}, h b l \mathrm{~A}, n h e \mathrm{BC}, p l c \mathrm{~A}, s p h$ and vip3A), encoding putative virulence factors was assessed by polymerase chain reaction (PCR) assays in a collection of 80 Brazilian B. cereus and B. thuringiensis strains (Zahner et al. 2005). Amplicons were detected for all of the sequences encoding factors suggested to play a role in infections of mammals. Interestingly, the majority of the sequences were detected more frequently in $B$. thuringiensis isolates than in B. cereus.

Multiple locus sequence typing (MLST) studies are based on detailed analysis of the nucleotides sequence of housekeeping genes. This approach has been extensively used in the identification of genetic variation within different groups of bacteria in order to develop evolutionary frameworks, to conduct epidemiological surveillance, for the identification of epidemic clones of anti- 
biotic resistant strains and to examine the evolution of virulence properties and population structure (Maiden et al. 1998, Priest et al. 2004, Helgason et al. 2004, Ko et al. 2004, Barker et al. 2005, de Filippis \& Vicente 2005, Hoffmaster et al. 2008). In this method, relationships among isolates are based on comparisons of allelic profiles: isolates sharing four or more alleles are considered as closely related sequence types (STs).

Different groups have provided evidence for associations between specific STs or lineages and the virulence potential of strains, exemplified by pathogenic $B$. cereus isolates from periodontal disease and emetic gastrointestinal illness that were associated with specific clonal groups (Helgason et al. 2000a, b, Barker et al. 2005, Ehling-Schulz et al. 2005, Vassileva et al. 2006, Hoffmaster 2008). Yet, the overall population structure of the $B$. cereus group is not clear due to the limited numbers of strains present in the data bank. It is generally accepted that there exists a necessity to expand the number housekeeping gene sequences deposited in the data bank in order to more clearly elucidate the existence of lineages or clonal complexes associated with specific diseases.

The first objective of the current study was to use MLST to determine the genetic relationships of 29 strains of B. cereus and B. thuringiensis with 18 of them isolated from different sources in Brazil and the remainder derived from a range of countries and sources. The second objective was to extend our earlier investigation in relation to the distribution of genes encoding putative virulence factors, specifically be, responsible for synthesis of the emetic toxin cereulide (Toh et al. 2004), cytK encoding necrotic enterotoxin (Fagerlund et al. 2004), in A encoding a metalloprotease of $B$. cereus (Gutmann \& Ellar 2000), the sequences pag, lef and cya located on the pXO1 plasmid of $B$. anthracis and the $B$. cereus plasmid $\mathrm{pBCX} 01$ and finally the gene cap encoding the capsular antigen of pXO2 (Jackson et al. 1998). The present work complements and enhances the previous study of Zahner et al. (2005) where the relationship between $B$. cereus and B. thuringiensis was investigated.

\section{MATERIALS AND METHODS}

Bacterial strains - Twenty-nine strains including $B$. cereus or B. thuringiensis and B. mycoides were included in this study (Table I). All these strains were originally present in Coleção de Culturas do Gênero Bacillus e Gêneros Correlatos - Bacillus Collection/Oswaldo Cruz Institute/Oswaldo Cruz Foundation. Details of each strain can be found in Zahner et al. (2005).

Molecular methods - Extraction and purification of genomic DNA has been described previously (Zahner et al. 2005). The presence the gene be, encoding emetic toxin, was examined by PCR as described by Toh et al. (2004) and be-PCR amplification products were sequenced. The $c y t \mathrm{~K}$ sequences were detected according to the protocol of Fagerlund et al. (2004), with the primers CytkF 5'-CCAACCCAGTTACCAGTTCC-3' and CytkR 5'-AACAGATATCGGTCAAAATGC-3' and the inA gene was amplified according to the methods of Gutmann and Ellar (2000). Nested-PCR for amplification of the structural genes of the entire complement of $B$. anthracis toxins found on pXO1 (pag, lef, cya) and the capsular antigen gene of $\mathrm{pXO} 2$ (cap) was carried out as described by Jackson et al. (1998). Details of primers used are provided in Table II. Strains showing a negative result for the be sequence by PCR assay were submitted to Southern blotting and hybridization with a be probe as previously reported (Zahner et al. 1998). Each PCR reaction was repeated at least three times to confirm reproducibility. This publication made use of the Bacillus cereus Multi Locus Sequence Type website (pubmlst.org/bcereus) developed by Jolley et al. (2004) analyzing the seven housekeeping genes ( $g l p, g m k, i l v D, p t a, p u r, p y c A$ and tpi) employing PCR conditions as described in the MLST database. PCR products from MLST genes and emetic toxin gene (be) were purified using the GFX-PCR DNA Kit (GE Healthcare Life Sciences). PCR fragments were sequenced in both directions, using the amplification primers to provide unambiguous sequence data, by use of the BigDye Ready Reaction mix (ABI Corp) and reaction products were analyzed on a Prism 3700 automated DNA analyser (ABI Corp). All the sequencing procedures were performed as published by Otto et al. (2008).

Data analysis - Sequencher 4.8 software (Genecodes) was used for assembly of contigs and determination of consensus sequences. The nucleotide sequences of each housekeeping gene were trimmed to the appropriate length (348-504 bp), as previously described (Jolley et al. 2004), and then queried to the MLST database. STs were assigned based on the combination of the seven alleles in a specific order. New alleles and allele sets were deposited in the Bacillus MLST database. Designation of new alleles and ST was determined by MLST database curators. A phylogenetic tree was constructed based on the multiple alignments of the concatenated sequences of the seven MLST genes through the neighbour-joining method using Mega 4.0 software (Tamura et al. 2007). Split decomposition analysis of allelic profiles was carried out with the SplitsTree 4.0 software (Huson 1998). Some known strains from the MLST Bacillus databank were included to assess the relationships of those isolates with the ones identified in our study.

\section{RESULTS}

Table I presents the distribution of virulence genes, variable-number of tandem repeats (VNTR) types, STs and origin of the Bacillus species examined.

The Figure represents phylogenetic relationships determined for the strains utilized in this study. It was observed that $B$. thuringiensis and $B$. cereus isolates were intermixed within the phylogenetic clusters and that no $B$. thuringiensis or $B$. cereus clustered with the $B$. anthracis group. Each of the strains belonged to a novel ST and occurred only once (STs 516-544) (Table I). The number of alleles per locus was: 16 ( $g l p)$, nine (gmk), 15 (ilvD), 18 (pta), 16 (pur), 15 (pycA) and 15 (tpi). The alleles glp13, gmK-8, ilvD-9, pta-11, pur-18, pycA-14 and tpi-7 were the most frequent per locus. No specific allele could be associated with either the origin of isolation or country. Analysis of the allelic diversity among the isolates result- 
s

昰

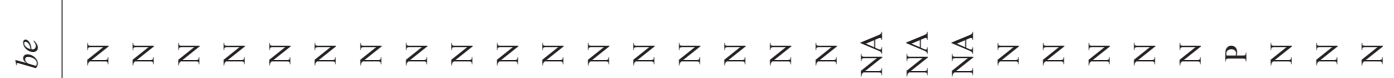

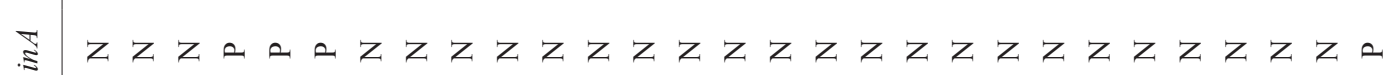

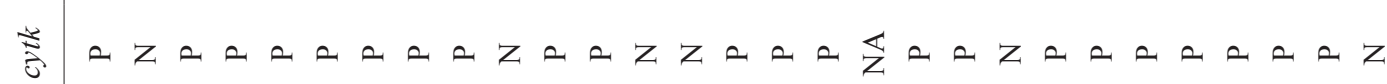

苨

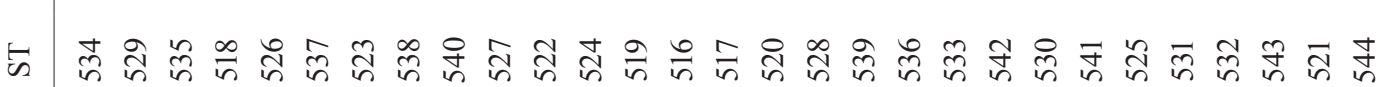

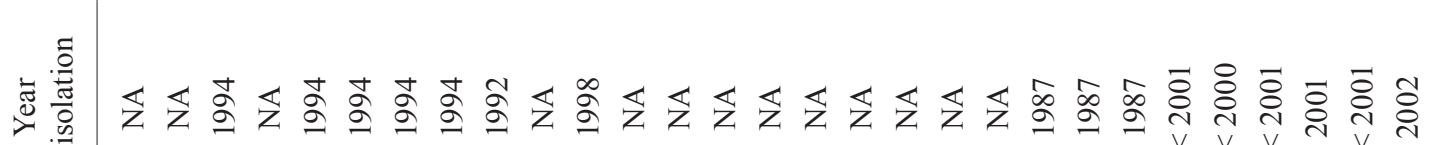
岁

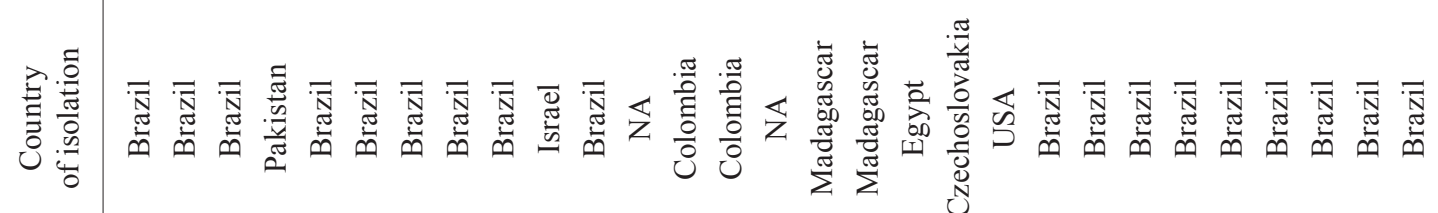

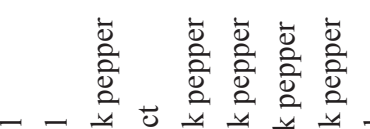

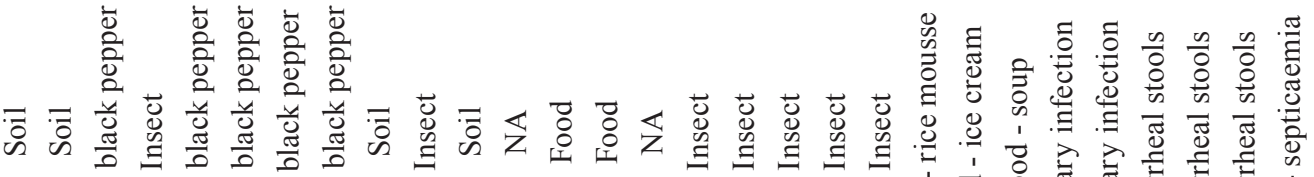

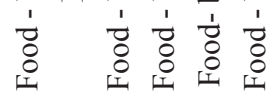

3

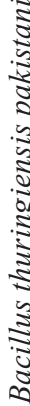

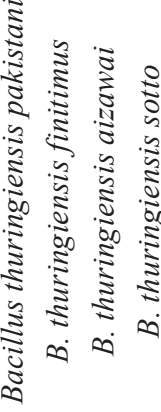

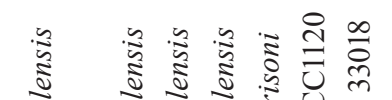


ed in the average number of alleles per locus of 14.8. A phylogenetic tree (Figure) was constructed from a single sequence of $2,838 \mathrm{bp}$ of concatenated loci sequences. The tree was derived using the novel isolates and included sequence data derived from previously characterized iso-

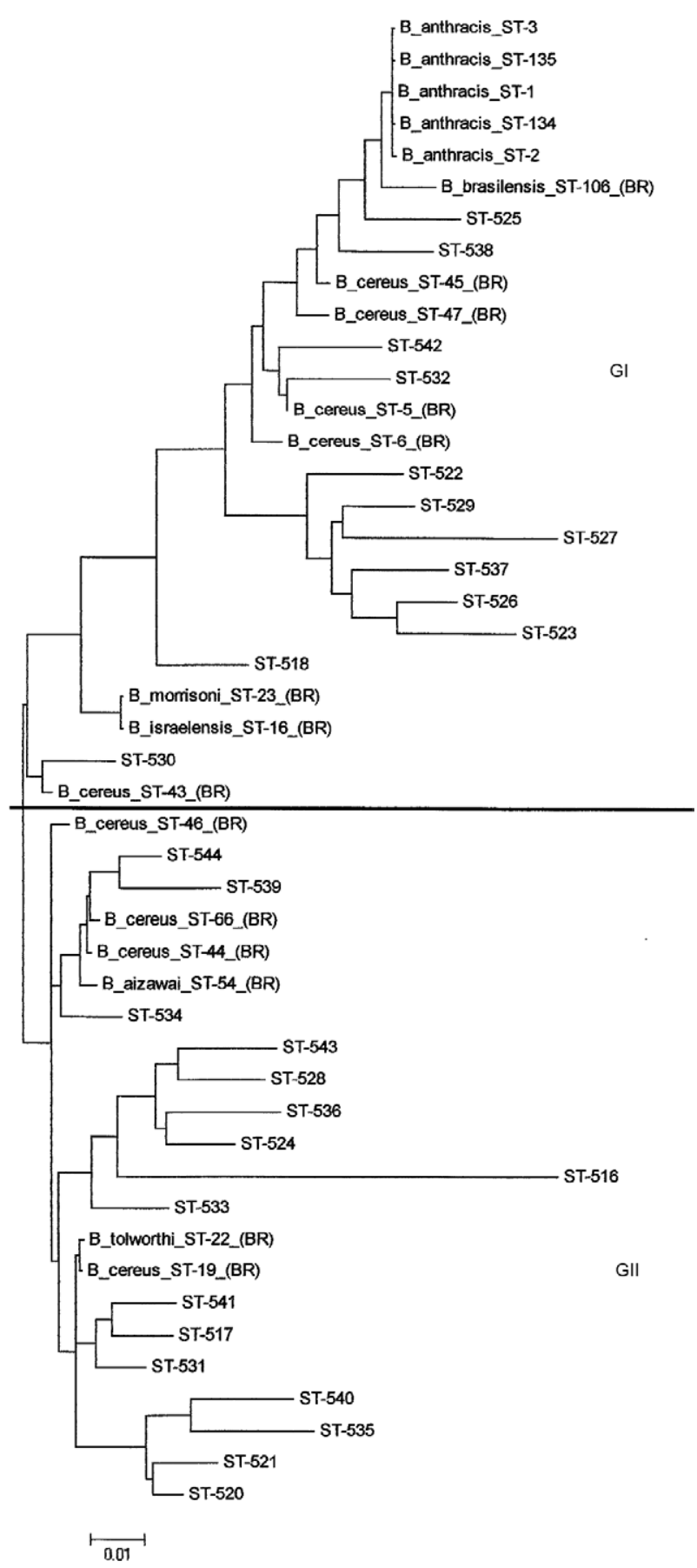

Relationships between novel isolates of this study and selected reference isolates derived from the multiple locus sequence typing $\mathrm{T}$ database using concatenated sequences from seven housekeeping alleles. The tree was constructed using the neighbour joining method. Clades and lineages are labelled as designated by Priest et al. (2004). The overall structure of the maximum-likelihood trees generated from concatenated sequences resulted in two major phylogenetic groups. ST: sequence type. lates stored in the Bacillus cereus Multi Locus Sequence Type website (pubmlst.org/bcereus). The overall structure of the maximum-likelihood tree generated from concatenated sequences resulted in two major phylogenetic groups (Figure): G1 (group 1), includes strains of clade 1, and G2 (group 2), includes strains pertaining to clade 2 as defined by Priest et al. (2004).

\section{DISCUSSION}

The first objective of the current study work was to increase the number of strains in the MLST database, in an attempt to more confidently assess if there are specific lineages or clonal complexes containing clinical isolates that might be associated with a specific illness or defined biological characteristics. For this reason we selected a range of $B$. cereus and $B$. thuringiensis strains isolated from a variety of environments and countries, with the majority (64.3\%) of them isolated in Brazil.

The study of Zahner et al. (1989) focused on population structure and diversity of the $B$. cereus and $B$. thuringiensis group based on the use of multilocus enzyme electrophoresis (MLEE) and concluded that the isolates examined represented a single species, it was also demonstrated that the $B$. cereus complex presented a high level of genetic diversity. Similar conclusions were reached from subsequent studies applying MLEE to evaluation of this group of bacteria (Helgason et al. 2000a, b, Villas-Boas et al. 2002). However, it is generally accepted that MLEE has limited applicability especially in relation to data analysis and the interchange of results between laboratories, while MLST provides some solutions to those shortcomings. The main advantages of MLST, over MLEE (the basis for MLST), are that DNA is more variable than encoded proteins (on account of primarily synonymous mutations) and fewer loci need to be examined in MLST. Furthermore, nucleotide sequence data are generic, definitive, readily compared among laboratories and easily analyzed by a range of phylogenetic and population genetic techniques. Such data can be made freely accessible via the internet, establishing virtual isolate collections that enable the rapid and accurate comparison of the genetic profiles of isolates obtained anywhere in the world (Maiden et al. 1998).

Different authors have used MLST in attempts to elucidate intra and inter $B$. cereus relationships. In order to characterize the population structure and epidemiology of B. cereus group, different MLST schemes have been developed: the Priest scheme (pubmlst.org/bcereus/), the Tourasse-Helgason scheme (mlstoslo.uio.no/) (spock.jouy.inra.fr/cgi-bin/bacilliMLSopen.cgi) (Sorokin et al. 2006), the Ko scheme (Ko et al. 2004) and the SuperCat scheme (Tourasse \& Kolstø 2008). Helgason et al. (2004) and Priest et al. (2004) using different MLST genes suggested a weakly clonal population structure for $B$. cereus. In this case, according to the authors, the evolutionary changes occurred via point mutations. Subsequently, Sorokin et al. (2006) studying localized populations of $B$. cereus group in soil suggested that $B$. cereus and $B$. thuringiensis are clonal. In addition, Hoffmaster et al. (2008) examined the phylogenetic diversity 
and relatedness of $B$. cereus clinical isolates present in a collection of $B$. cereus recovered along 50 years and observed that those isolates were phylogenetically diverse.

Among the different MLST schemes we opted to use the one described by Priest et al. (2004) because of the advantage of allele and ST number facilitating immediate identification of the strain, a feature which is lacking in other methods such as the SuperCat scheme. In addition, this databank holds sequences produced from numerous Brazilian isolates facilitating strain comparison. Corroborating previous observations, it was determined that the $B$. thuringiensis and $B$. cereus isolates were intermixed within the phylogenetic clusters. However, no $B$. thuringiensis or B. cereus clustered within the $B$. anthracis group. The overall topology of the tree also provided support to previous data (Hoffmaster et al. 2008), showing that the $B$. cereus complex population is clonal with clinical and environmental isolates emerging from different phylogenetic positions.
The 29 test strains were recovered from different sources including soil, insects, human infections, foodstuffs and a lethal case of avian septicaemia; with the majority isolated in Brazil. Each of the strains analyzed belonged to a novel ST and occurred only once (STs 516-544) (Table I), demonstrating that, as suggested by other authors, MLST is a useful and powerful tool for molecular epidemiology (Maiden et al. 1998 , Sorokin et al. 2006, Ibarz \& Maiden 2009). Analysis of the allelic diversity among the isolates resulted in the average number of alleles per locus of 14.8, which is relatively low in comparison to the values of 19.9, 33.6 and 30.5 which were obtained by other authors (Helgason et al. 2004, Priest et al. 2004, Hoffmaster et al. 2008). No specific allele could be associated with either the origin of isolation or country. Given the fact that the strains were obtained from a variety of sources, the observed values strongly indicated the clonal aspect of evolution within the $B$. cereus complex.

TABLE II

Sequences of DNA oligonucleotides used as primers in polymerase chain reaction for detection of virulence genes

\begin{tabular}{|c|c|c|c|}
\hline Target & Primer sequence 5 '-3' & $\begin{array}{l}\text { Product size } \\
\text { (bp) }\end{array}$ & Reference \\
\hline \multirow[t]{2}{*}{ pag } & F CCAGACCGTGACAATGATG & 508 & Jackson et al. (1998) \\
\hline & R CAAGTTCTTTCCCCTGCTA & & \\
\hline \multirow[t]{2}{*}{$\operatorname{pag}^{a}$} & F CGAAAAGGTTACAGGACGG & 409 & Jackson et al. (1998) \\
\hline & R CAAGTTCTTTCCCCTGCTA & & \\
\hline \multirow[t]{2}{*}{ lef } & F GGTGCGGATTTAGTTGATTC & 851 & Jackson et al. (1998) \\
\hline & R CGCTTCATTTGTTCTCCC & & \\
\hline \multirow[t]{2}{*}{$l e f^{a}$} & F GAAACATCGGTCTGGAAAT & 403 & Jackson et al. (1998) \\
\hline & R CCCTTTTGAATGAACTTGC & & \\
\hline \multirow[t]{2}{*}{ cya } & F GCGATGAAAACAACGAAGTA & 720 & Jackson et al. (1998) \\
\hline & R TCGTCTTTGTCGCCACTATC & & \\
\hline$c y a^{a}$ & F CATTAGAAAAGCAAAAAGGTC & 186 & Jackson et al. (1998) \\
\hline \multirow[t]{2}{*}{ capA } & R TCATTATCTTGCTCTGTGCC & & \\
\hline & F CAGAAGCAGTAGCACCAGTAA & 397 & Jackson et al. (1998) \\
\hline \multirow[t]{2}{*}{$\operatorname{cap\mathrm {A}^{a}}$} & R ATTTTCACCAGCACCCAC & & \\
\hline & F TGACGATGGTTGGTGACA & 302 & Jackson et al. (1998) \\
\hline \multirow[t]{2}{*}{$\operatorname{cap} \mathrm{B}$} & R CCTTATTGTATCTTTAGTTCCC & & \\
\hline & F CTGACCAATCTAAGCCTGC & 221 & Jackson et al. (1998) \\
\hline \multirow[t]{2}{*}{$\operatorname{cap} \mathrm{C}$} & R TCGTTTCTCCAATCGCAAT & & \\
\hline & F GTACCTGGTTATTTAGCACTCG & 208 & Jackson et al. (1998) \\
\hline \multirow[t]{2}{*}{$b e$} & R ATCTCAAATGGCATAACAGG & & \\
\hline & F ACTTAGATGATGCAAGACTG & 850 & Toh et al. (2004) \\
\hline \multirow[t]{2}{*}{$\operatorname{in} \mathrm{A}$} & R TTCATAGGATTGACGAATTTT & & \\
\hline & F TGAGCCATTCGCGTTAGAGG & 529 & Gutmann and Ellar (2000) \\
\hline \multirow[t]{3}{*}{$c y t \mathrm{~K}$} & R ATATTCATGCGCGTTTACACC & & \\
\hline & F CCAACCCAGTTACCAGTTCC & 810 & Fagerlund et al. (2004) \\
\hline & R AACAGATATCGGTCAAAATGC & & \\
\hline
\end{tabular}

$a$ : internal primers used in nested polymerase chain reaction. 
The phylogenetic tree (Figure) demonstrated that the strains examined in this study were not monophyletic by analysis of any specific housekeeping gene or characteristic previously known or described herein; instead, they were distributed among the Bacillus group G1 and G2 and in different previously defined lineages (Priest et al. 2004).

Analysis was undertaken to investigate if any of the examined virulence factors could be considered as characteristic for a specific phylogenetic lineage. Our results demonstrated that there was no clear association in relation to source of isolation or to genotypic profile based on the presence or absence of putative virulence genes (Table I), confirming the findings of an earlier but less extensive genotypic examination of these isolates (Zahner et al. 2005). Furthermore, B. cereus and B. thuringiensis were shown to be distributed among several lineages, although unique species could be associated with specific STs, such as 522 and 529, 526 and 537, all B. thuringiensis isolated from soil and food, respectively, or STs 532 (clinical) and 542 (food), both B. cereus, ascribed to lineage Cereus I (Priest et al. 2004). The B. cereus isolate LFB 1181(ST-544) was recovered from an outbreak of fatal septicaemia in captive birds in Brazil. Interestingly, when this strain was tested for cytotoxicity towards Chinese hamster ovary cells it presented the highest level (titre 1:256) of toxicity among the test isolates (data not shown). Moreover, isolate LFB1181 was the only strain of $B$. cereus which was recorded as inhA PCR positive, suggesting a possible correlation between this enzyme and the disease outbreak in birds. In this context, Guillemet et al. (2010) suggested that the InhA metalloproteases of $B$. cereus are important virulence factors that may allow bacteria to counteract the host immune system. Intriguingly, this sequence was also detected in two Brazilian isolates of $B$. thuringiensis (serotypes aizawai and israelensis), both from black pepper, and a strain of $B$. thuringiensis (serotype sotto) isolated from an insect in Pakistan. The presence of sequences encoding this protease in environmental isolates, generally considered as non-pathogenic for mammals, suggests that as noted for other putative virulence factors $(b c e \mathrm{~T}, h b l \mathrm{~A}, n h e \mathrm{BC}$, $p l c \mathrm{~A}$ and $s p h$ ), the role of this protein is not primarily the establishment or maintenance of infection in warm blooded hosts (Zahner et al. 2005).

Ehling-Schulz et al. (2005) using data based on MLST, reported that cereulide-producing emetic $B$. cereus presented a clonal population structure, forming a highly clonal complex. The panel of isolates used in this study did not include any strains involved in food poisoning, but interestingly the presence of the sequence be, which in emetic strains is carried on mega plasmids of more than 200 kilobase pairs and is involved in cereulide synthesis, was detected in LFB598 (ST-532) by PCR (Table I). The be derived amplicon of the positive strain LFB598 (ST532) were sequenced and BLAST analysis indicated that it shared 96\% homology with be gene sequences deposited in the database. The strain LFB598 was isolated from a Brazilian stool sample and was placed in GII. Yet, it should be stressed that no tests were performed to determine if the PCR positive isolate expressed functional cereulide toxin.
The presence of sequences encoding the tripartite toxin and poly-D-glutamate capsule of $B$. anthracis was investigated via PCR however no isolates, including the avian pathogenic strain LFB1181, generated amplicons for any of the genes examined. The $B$. cereus isolate which was placed closest to $B$. anthracis in our comparative analysis (Figure) was strain LFB585 (ST-525), a Brazilian isolate recovered from a urinary tract infection (Table I). The genetic profile of this strain, in terms of putative virulence factors, as previously demonstrated (Zahner et al. 2005) and enhanced by data from the present work, indicates that the strain is PCR positive for $c y t K, h b l$, nheBC, bceT, $s p h$, but there was no evidence by PCR or nested PCR for $B$. anthracis $\mathrm{pXO} 1$ and $\mathrm{pXO} 2$ virulence genes. The proximity of ST-525 with B. anthracis adds some support to the hypothesis that $B$. cereus isolates that cluster closely with $B$. anthracis are associated with clinical cases (Helgason et al. 2000a, b, Hill et al. 2004). Yet on the other hand, an additional clinical isolate recovered from faeces (ST-543) and placed in GII was distant from B. anthracis (Figure). This apparent contradiction highlights the aforementioned shortcomings of the existing MLST database.

Although the majority of our B. cereus samples were either environmental or food associated, we also analyzed a number of clinical isolates. In the cladrogram, using strains already present in the MLST database (Figure), numerous environmental strains were firmly placed in the same group as our clinical isolates. Thus, it could be suggested that all B. cereus sensu latu posses pathogenic potential, but what triggers the pathogenicity is still not known. Clearly the mere presence of sequences encoding the putative virulence factors examined in this study is not sufficient to definitively confer pathogenicity. Hence, it is worth considering the observation of Hoffmaster et al. (2004), who stated that the isolation of B. cereus in a clinical laboratory is frequently considered as contamination, with strains commonly being discarded rather than followed up with further characterization and reporting. Consequently, the analysis of clinical isolates is often inadequate resulting in an underestimation of both the frequency and significance of non-gastrointestinal infections caused by $B$. cereus and possibly other members of the $B$. cereus group.

Despite the sophistication of the methodology, the inability of MLST to link virulence with genotype was further demonstrated by the observation that strain LFB1181 (ST-544) was firmly placed in the same clade as strains isolated from a variety of environmental sources and which demonstrated distinct genotypic characteristics (Table I). This observation adds support to the notion that the putative virulence factors are randomly distributed among $B$. cereus and $B$. thuringiensis and that a simple assessment for the presence of these factors will be unable to confidently predict the virulence of members of the B. cereus group (Guttman \& Ellar 2000, Zahner et al. 2005). Support for this statement, in the context of food poisoning, was recently provided by the work of Guinebretière et al. (2010), who demonstrated that the ability of B. cereus group strains to cause food poisoning varies according to phylogenetic affiliation (groups I-VII) rather than species affiliation. 
In light of their abundance and extensive polymorphism, VNTR have proven to be fundamental genetic markers in bacterial population and/or phylogenetic analysis. Analysis based on VNTR compares the strain-specific numbers of repeats of short DNA sequences at different positions of the bacterial genome and provides functional and evolutionary information concerning genetic relationships in microbial species (Van Belkum 1999). Moreover, in the case of some highly clonal bacterial species, e.g. Acinetobacter baumanni, VTNR analysis may serve as a powerful discriminatory method for the identification of distinct populations of highly similar isolates associated with disease (Turton et al. 2011). The 29 test strains were previously characterized by VNTR analysis, using a variable region of the $v r r$ A open reading frame as the target and were ascribed to five different fragment length categories (Zahner et al. 2005). A comparison of VNTR profile and STs was performed in the present study (Table I). In contrast to the analysis in relation to the presence/absence of putative virulence factors, a correlation between VNTR profile and MLST was suggested. Specifically $63 \%$ of strains identified as VNTR 4 or 5 were placed in GI, while in GII $73 \%$ presented the VNTR profiles 1, 2 or 3. However, once again this apparent association between genetic features was unable to provide a means of confidently discriminating between clinical and environmental members of the $B$. cereus complex.

With the possible exceptions of emetic and periodontal isolates of B. cereus (Ehling-Schulz et al. 2005, Vassileva et al. 2006, Hoffmaster et al. 2008) and the plasmid encoded virulence factors of $B$. anthracis, the use of genetic characterization, including the data reported in the current study, has largely failed to explain the basis for pathogenicity of members of the $B$. cereus complex. Recent proteomic analysis (Claire et al. 2010) has served to increase the repertoire of putative virulence factors expressed by B. cereus, with 31 novel virulence related molecules identified in the secretome. The expression of these factors by other members of the $B$. cereus complex has yet to be investigated. However, it is becoming increasingly apparent that molecular characterization in the absence of functional analysis of putative virulence gene expression is unlikely to clarify the mechanisms which allow a given isolate to cause disease. On the other hand, our application of MLST has served to reinforce the notion that $B$. cereus and $B$. thuringiensis should be considered as the same species (Helgason et al. 2000a, b, Anderson et al. 2005, Zahner et al. 2005, Han et al. 2006).

\section{REFERENCES}

Anderson I, Sorokin A, Kapatral V, Reznik G, Bhattacharya A, Mikhailova N, Burd H, Joukov V, Kaznadzey D, Walunas T, MarkD'Souza, Larsen N, Pusch G, Liolios K, Grechkin Y, Lapidus A, Goltsman E, Chu L, Fonstein M, Ehrlich SD, Overbeek $\mathrm{R}$, Kyrpides N, Ivanova N 2005. Comparative genome analysis of Bacillus cereus group genomes with Bacillus subtilis. FEMS Microbiol Lett 250: 175-184.

Barker M, Thakker B, Priest FG 2005. Multilocus sequence typing reveals that Bacillus cereus strains isolated from clinical infections have distinct phylogenetic origins. FEMS Microbiol Lett 245: 179-184.
Clair GS, Roussi S, Armengaud J, Duport C 2010. Expanding the known repertoire of virulence factors produced by Bacillus cereus through early secretome profiling in three redox conditions. Mol Cell Proteomics 9: 1486-1498.

de Filippis I, Vicente AC 2005. Multilocus sequence typing and repetitive element-based polymerase chain reaction analysis of Neisseria meningitides isolates in Brazil reveal the emergence of 11 new sequence types genetically related to the ST-32 and ST-41/44 complexes and high prevalence of strains related to hypervirulent lineages. Diagn Microbiol Infect Dis 53: 161-167.

Ehling-Schulz M, Svensson B, Guinebretiere MH, Lindback T, Andersson M, Schulz A, Fricker M, Christiansson A, Granum PE, Martlbauer E, Nguyen-The C, Salkinoja-Salonen M, Scherer S 2005. Emetic toxin formation of Bacillus cereus is restricted to a single evolutionary lineage of closely related strains. Microbiology 151: 183-197.

Fagerlund A, Ween O, Lund T, Hardy SP, Granum PE 2004. Genetic and functional analysis of the cytK family of genes in Bacillus cereus. Microbiology 150: 2689-2697.

Guillemet E, Cadot C, Tran SL, Guinebretière MH, Lereclus D, Ramarao N 2010. The InA metalloproteases of Bacillus cereus concomitantly to virulence. J Bacteriol 192: 286-294.

Guinebretière MH, Velge P, Couver O, Carlin F, Debuyser ML, Nguyen C 2010. Ability of Bacillus cereus group strains to cause food poisoning varies according to phylogenetic affiliation (groups I to VII) rather than species affiliation. J Clin Micro 48: 3388-3391.

Guttman DM, Ellar DJ 2000. Phenotypic and genotypic comparisons of 23 strains from the Bacillus cereus complex for a selection of known and putative $B$. thuringensis virulence factors. FEMS Microbiol Lett 188: 7-13.

Han CS, Xie G, Challacombe JF, Alther MR, Bhotika SS, Brown N, Bruce D, Campbell CS, Campbell M L, Chen J, Chertkov O, Cleland C, Dimitrijevic M, Dogget NA, Fawcett JJ, Glavina T, Goodwin LA, Green LD, Hill KK, Hitchcock P, Jackson PJ, Keim P, Kevalramani AR, Longmire J, Lucas S, Malfatti S, McMurry K, Meincke LJ, Misra M, Moseman BL, Mundt L, Munk AC, Okinaka RT, Parson-Quintana B, Reilly LP, Richardson P, Robinson DL, Rubin E, Saunders E, Tapia R, Tesmer JG, Thayer N,Thompson LS, Tice H, Ticknor LO, Wills PL, Brettin TS, Gilna P 2006. Pathogenomic sequence analysis of Bacillus cereus and Bacillus thuringiensis isolates closely related to Bacillus anthracis. J Bacteriol 188: 1662-1833.

Helgason E, Caugant DA, Olsen I, Kolsto AB 2000a. Genetic structure of population of Bacillus cereus and B. thuringiensis isolates associated with periodontitis and other human infections. $J$ Clin Microbiol 38: 1615-1622.

Helgason E, Økstad OA, Caugant DA, Johansen HA, Fouet A, Mock M, Hegna I, Kolstø A-B 2000b. Bacillus anthracis, Bacillus cere$u$ and Bacillus thuringiensis. One species on the basis of genetic evidence. Appl Environ Microbiol 66: 2627-2630.

Helgason E, Tourasse NJ, Meisal R, Caugant DA, Kolstø AB 2004. Multilocus sequence typing scheme for bacteria of the Bacillus cereus group. Appl Environ Microbiol 70: 191-201.

Hill K, Ticknor LO, Okinaka RT, Asay M, Blair H, Bliss KA, Laker M, Pardington PE, Richardson P, Tonks M, Beecher DJ, Kemp JD, Kolsto AB, Lee WAC, Keim P, Jackson PJ 2004. Fluorescent amplified fragment length polymorphism analysis of Bacillus anthracis, Bacillus cereus and Bacillus thuringiensis isolates. Appl Environ Microbiol 70: 1068-1080.

Hoffmaster AR, Novak RT, Marston CK, Gee JE, Helsel L, Pruckler JM, Wilkins PP 2008. Genetic diversity of clinical isolates of $B a$ cillus cereus using multilocus sequence typing. BMC 6: 191. 
Hoffmaster AR, Ravel J, Rasko DA, Chapman GD, Chute MD, Marston CK, Barun KD, Sacchi CT, Fitzgerald C, Mayer LW, Maiden MC, Priest FG, Barker M, Jiang L, Cer RZ, Rilstone J, Peterson SN, Weyant RS, Galloway DR, Read TD, Popovic T, Fraser CM 2004. Identification of anthrax toxin genes in a Bacillus cereus associated with an illness resembling inhalation anthrax. Proc Natl Acad Sci USA 101: 8449-8454.

Huson DH 1998. SplitsTree: a program for analyzing and visualizing evolutionary data. Bioinformatics 14: 68-73.

Ibarz PAB, Maiden MC 2009. Multilocus sequence typing. Method Mol Biol 551: 129-140.

Jackson PJ, Hugh-Jones ME, Adair DM, Green G, Hill KK, Kuske CR, Grinberg LM, Abramova FA, Keim P 1998. PCR analysis of tissue samples from the 1979 Sverdlovsk anthrax victims: the presence of multiple Bacillus anthracis strains in different victims. Proc Natl Acad Sci USA 95: 1224-1229.

Jensen GB, Hansen BM, Eilenberg J, Mahillon J 2003. The hidden lifestyles of Bacillus cereus and relatives. Environ Microbiol 5: 631-640.

Jolley KA, Chan MS, Maiden MC 2004. MlstdbNet-distributed multi-locus sequence typing (MLST) databases. BMC Bioinformatics 5: 86 .

Ko KS, Kim JW, Kim JM, Kim W, Chung SI, Kim IJ, Kook YH 2004. Population structure of the Bacillus cereus group as determined by sequence analysis of six housekeeping genes and the $p l c \mathrm{R}$ gene. Infect Immun 72: 5253-5261.

Lund T, de Buyser ML, Granum PE 2000. A new cytotoxin from Bacillus cereus that may cause necrotic enteritis. Mol Microbiol 38: $254-261$.

Mahler H, Pasi A, Kramer JM, Schulte P, Scoging AC, Bär W, Krähenbühl S 1997. Fulminant liver failure in association with the emetic toxin of Bacillus cereus. New Engl J Med 336: 1142-1148.

Maiden MC, Bygraves JA, Feil E, Morelli G, Russell JE, Urwin R, Zhang Q, Zhou J, Zurth K, Caugant DA, Feavers IM, Achtman M, Spratt BG 1998. Multilocus sequence typing: a portable approach to the identification of clones within populations of pathogenic microorganisms. Proc Natl Acad Sci USA 95: 3140-3145.

Otto TD, Vasconcellos EA, Gomes LHF, Moreira AS, Degrave WM, Mendonça-Lima L, Alves-Ferreira M 2008. 15ChromaPipe: a pipeline for analysis, quality control and management for a DNA sequencing facility. Genet Mol Res 7: 861-871.
Priest FG, Barker M, Baillie L, Holmes EC, Maiden MC 2004. Population structure and evolution of the Bacillus cereus group. J Bacteriol 186: 7959-7970.

Sorokin A, Candelon B, Guilloux K, Galleron N, Wackerow-Kouzova N, Ehrlich SD, Bourguet D, Sanchis V 2006. Multiple-locus sequence typing analysis of Bacillus cereus and Bacillus thuringiensis reveals separate clustering and a distinct population structure of psychotropic strains. Appl Environ Microbiol 72: 1569-1578.

Tamura K, Dudley J, Nei Kumar S 2007. MEGA4: Molecular Evolutionary Genetics Analysis (MEGA) software version 4.0. Mol Biol Evol 24: 1596-1599.

Toh M, Moffitt MC, Henrichsen L, Raftery M, Barrow K, Cox JM, Marquis CP, Neilan BA 2004. Cereulide, the emetic toxin of $B a-$ cillus cereus, is putatively a product of nonribosomal peptide synthesis. J Appl Microbiol 97: 992-1000.

Tourasse NJ, Kolstø AB 2008. SuperCAT: a supertree database for combined and integrative multilocus sequence typing analysis of the Bacillus cereus group of bacteria (including B. cereus, B. anthracis and B. thuringiensis). Nucl Acids Res 36: 461-468.

Turton JF, Baddal B, Perry C 2011. Use of the accessory genome for characterization and typing of Acinetobacter baumannii. J Clin Micro 49: 1260-1266.

Van Belkum A 1999. Short sequence repeats in microbial pathogenesis and evolution. Cell Mol Life Sci 56: 729-734.

Vassileva M, Torii K, Oshimoto M, Okamoto A, Agata N, Yamada K, Hasegawa T, Ohta M 2006. Phylogenetic analysis of Bacillus cereus isolates from severe systemic infections using multilocus sequence typing scheme. Microbiol Immunol 50: 743-749.

Villas-Boas G, Sanchis V, Lereclus D, Lemos MVF, Bourguet D 2002. Genetic differentiation between sympatric populations of Bacillus cereus and Bacillus thuringiensis. Appl Environ Microbiol 68: 1414-1424.

Zahner V, Cabral DA, Regua-Mangia AH, Rabinovitch L, Moreau G, McIntosh D 2005. Distribution of genes encoding putative virulence factors and fragment length polymorphism in the vrrA gene among Brazilian isolates of Bacillus cereus and Bacillus thuringiensis. Appl Environ Microbiol 71: 8107-8114.

Zahner V, Momen H, Priest FG 1998. Serotype H5aH5b is a major clone within mosquito-pathogenic strains of Bacillus sphaericus. Sys Appl Microbiol 21: 162-170.

Zahner V, Momen H, Salles CA, Rabinovitch L 1989. A comparative study of enzyme variation in Bacillus cereus and Bacillus thuringiensis. J Appl Bacteriol 67: 275-282. 